\title{
Liver Tumor Detection in CT Images by Adaptive Contrast Enhancement and the EM/MPM Algorithm
}

\author{
Yu Masuda et al.* \\ Department of Science and Engineering, \\ Ritsumeikan Univesity, Shiga, \\ Japan
}

\section{Introduction}

Liver cancer is considered one of the major causes of death in humans [1]. Early detection of tumors is essential for increasing the survival chances of patients. Recent advancements in medical imaging modalities have enabled the acquisition of high-resolution CT datasets, and thus, allowing physicians to identify both small and large tumors by manual visual inspection. Owing to the large number of images in medical datasets, it is difficult to manually analyze all images, and useful diagnostic information may be overlooked. Moreover, the diagnoses are mainly based on the physician's subjective evaluation and are dependent on the physician's experience. Therefore, computer assisted diagnosis (CAD) and computer assisted surgery have become one of the major research subjects.

Until now, many methods have been proposed for tumor detection and segmentation in liver CT images. These methods can be classified as semi-automatic [2][3] and automatic [4][5]. Smeets et al. have proposed a semi-automatic level set method, which combines a spiral scanning technique with supervised fuzzy pixel classification [2]. Mala et al. employed wavelet-based texture features in order to train a neural network for use in tumor detection [4]. In the method proposed by Park et al. [5], the voxels representing the liver vessels are removed from liver images and a bimodal histogram is assumed for the intensity distribution of the liver and tumors. The optimal threshold to segment tumors is determined by a "mixture probability density" algorithm. In our previous study [6], we proposed tumor detection [7], which is a technique combining the expectation maximization algorithm and a three-dimensional region of interest (ROI) detection method. However, if the image contrast is low, it is difficult to accurately remove vessels from the image. All the above-mentioned methods can locate tumors that are sufficiently large and have distinct boundaries. Semiautomatic approaches for handling a large number of tumors would need extensive user interactions, and therefore are error prone and tedious.

\footnotetext{
* Tomoko Tateyama ${ }^{1}$, Wei Xiong'2, Jiayin Zhou², Makoto Wakamiya ${ }^{3}$, Syuzo Kanasaki³, Akira Furukawa ${ }^{3}$ and Yen Wei Chen ${ }^{1}$

1 Department of Science and Engineering, Ritsumeikan Univesity, Shiga, Japan,

2 Institute for Infocomm Research, Singapore,

3 Shiga University of Medical Science, Shiga, Japan.
} 
We propose a new method for detecting tumors in CT images. Our method is based on adaptive contrast enhancement and the expectation maximization / maximization of the posterior marginal (EM/MPM) algorithm. User interaction is not required and both large and small tumors can be accurately found. Compared with our previously reported method [6], the newly proposed method is also suitable for images with poor contrasts. We describe the method in Sections 2-6 and present the experimental results in Section 7, followed by our conclusions.

\section{Overview of the proposed method}

Our method is composed of seven steps: (1) read the CT images; (2) extract the liver region using a well-established liver region segmentation program [8]; (3) smooth out the noise from the CT images; (4) enhance the CT image contrast by using probability density functions (PDFs) estimated from the training data; (5) remove vessels by applying Maximum likelihood method; (6) detect tumor candidates by employing the EM/MPM algorithm; and (7) detect and segment the tumor regions by using a shape filter.

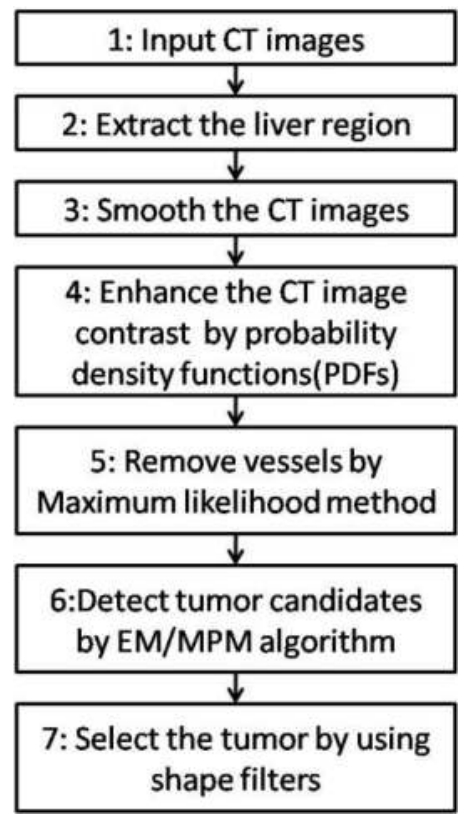

Fig. 1. The flowchart of our proposed method for tumor detections

\section{Contrast enhancement}

As tumor detection is mainly based on the intensity of CT images, the contrast of the images is very important. Two typical histograms of CT images having high and low contrasts are shown in Figs. 2(a) and 2(b), respectively. As shown in Fig.2(a), if the contrast of CT images is high, the tumor is in a different intensity range (left small peak) with the liver (right large peak) and the tumor can be easily detected by the intensity threshold, while if the contrast of 
CT images is low, the tumor is in the same narrow intensity range as the liver as shown in Fig.2(b) and it is difficult to detect the tumor from the liver volume. Density value of all objects is in a narrow range as shown in Fig.2(b). So we have to enhance the contrast of CT images as a preprocessing.

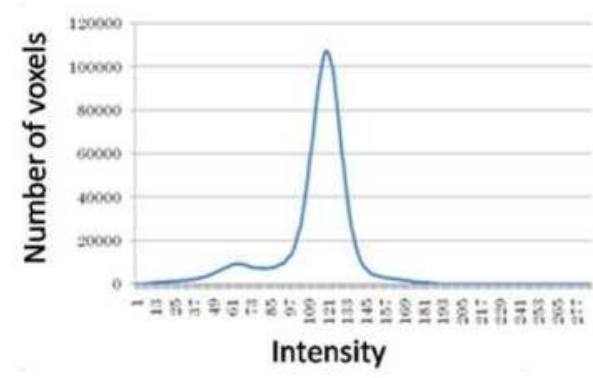

(a)

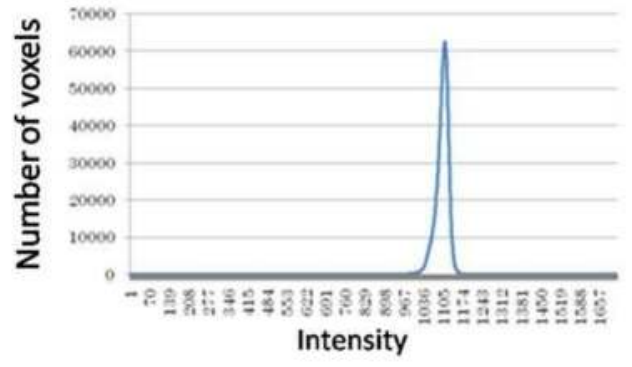

(b)

Fig. 2. Histograms with (a) high (b) low contrasts

A piecewise linear histogram transformation is usually employed to enhance the intensity contrast. However, it is difficult to determine a fixed set of lower and upper limits of the transformation slope for all images because they are data dependent. In this study, we automatically and adaptively determine the parameters from each image. In liver CT images, there are three classes of tissues: tumor, healthy liver, and vessels. First, sample voxels of the three classes are manually selected from the training data. The intensity PDFs of each class is estimated.

We also compute their mean values $\left(\mu_{\text {tumor }}^{A}, \mu_{\text {liver }}^{A}, \mu_{\text {vessel }}^{A}\right)$, their standard derivations $\left(\sigma_{\text {tumor }}\right.$, $\left.\sigma_{\text {liver }}, \sigma_{\text {vessel }}\right)$, and $M^{A} . M^{A}$ is the intensity value which has the highest probability in the liver class. The mean values have the property of $\mu_{\text {tumor }}^{A}<\mu_{\text {liver }}^{A}<\mu_{\text {vessel }}^{A}$. In Fig. 3(a), the three curves, from left to right, represent the PDFs of the tumor, the (healthy) liver, and the vessel classes. The mean and standard deviation values may differ among images, but the mean value of the (healthy) liver is always larger than that of the tumor and smaller than that of the vessels. This can be used for the classification of the three classes. The pattern of these three curves is called Curve Pattern A.

Given a new image and its segmented liver volume, we first compute the intensity histogram of all voxels in the liver volume. We find the intensity value $M^{B}$, which has the most component in the liver class, and assume that $M^{B}$ corresponds to the probability density peak of the class of healthy liver tissues in the new liver volume. Such an assumption is viable because the healthy tissues normally dominate the volume.

As a result, the mean values of the tumor and vessels are estimated as $\mu_{\text {tumor }}=M^{B}-M^{A}+\mu_{\text {tumor }}^{A}$ and $\mu_{\text {vessel }}=M^{B}-M^{A}+\mu_{\text {vessel }}^{A}$, respectively. Now, we set the lower limit as $T_{\min }=\mu_{\text {tumor }}-3 \sigma_{\text {tumor }}$ and the upper limit as $T_{\max }=\mu_{\text {vessel }}+3 \sigma_{\text {vessel }}$. The intensity transform formula for the range $0-255$ is given in Eq. (1). Using the formula, the intensity histograms of the liver images are re-estimated [Fig. 3(c)] for later use. 


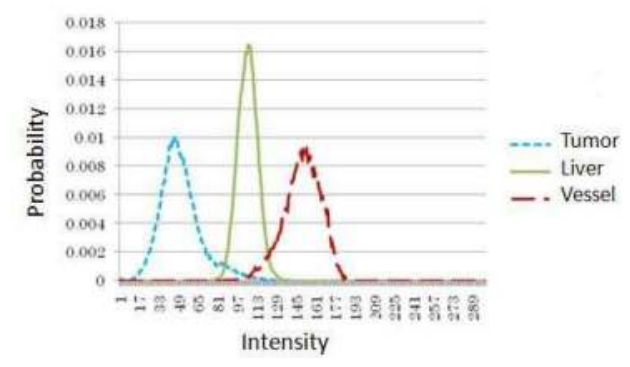

(a)

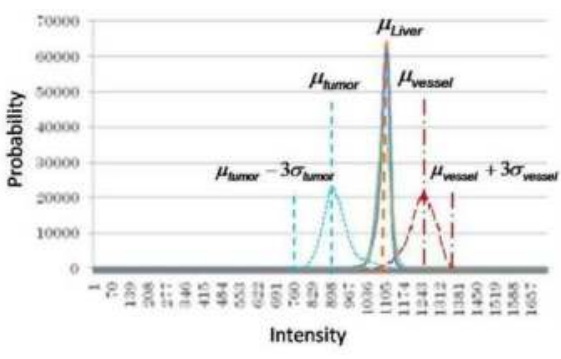

(b)

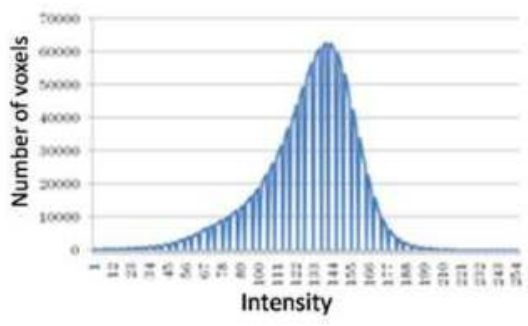

(c)

Fig. 3. (a) Estimated intensity PDFs for tumor (curve on the left), liver (curve in the center), and vessel (curve on the right); (b) Overlap the Curve Pattern A to the new image's liver volume histogram; (c) Histogram obtained after our histogram transformation

$$
\begin{aligned}
& \left\{\begin{array}{cc}
\frac{\left(x_{i}-T_{\min }\right)}{\left(T_{\max }-T_{\min }\right)}(255-1)+1 & \text { if }\left(T_{\min } \leq x_{i} \leq T_{\max }\right) \\
0 & \text { if }\left(x_{i}<T_{\min } \cup x_{i}>T_{\max }\right)
\end{array}\right. \\
& T_{\min }: \mu_{\text {tumor }}-3 \sigma, T_{\max }: \mu_{\text {vessel }}+3 \sigma
\end{aligned}
$$

\section{Removement of vessels by applying Maximum likelihood method}

Before tumor detection step, we first remove vessels from CT images. In the conventional method [5], as the intensity of vessels is higher than those of health liver tissues and tumor tissues, intensity threshold method is used to remove vessels. We classify the CT volume into 3 classes by using Maximum likelihood method. And then, voxels of the class with the highest mean are removed as vessels. After this process, CT images only include tumor and healthy liver tissues. The tumor detection problem can be simplified as a 2-class classification problem. This process will also significantly reduce the detection time.

\section{Tumor candidate detection by using EM/MPM algorithm}

To extract tumor candidate, we used the EM/MPM algorithm [9]. It is based on a Bayesian framework that assumes a Gaussian mixture to model intensity distribution and concurrently estimates both the labels of the voxels and the model parameters. In MPM, the 
cost function is defined to minimize the total number of misclassified voxels. It can be proved that minimization of the cost function is equivalent to maximization of the posterior marginal probability of the label fields (Eq. 2) [10].

$$
\hat{x}_{s_{M P M}}=\arg \max _{x} P_{X_{s} \mid Y}\left(x_{s} \mid y, \theta\right)=\arg \max _{x} \sum_{X \in \Omega_{k, s}} P_{X \mid Y}(x \mid y, \theta)
$$

In Eq. (2), $x, y$, and $\theta$ are the label vector, feature vector, and model parameters, respectively, $s$ is a pixel, $k$ is the label of pixel $s$, and $\Omega$ refers to all possible labels of the image in which the label of pixel $s$ equals $k$. The posterior probability is composed of two factors, namely the likelihood function and the prior probability. The likelihood function is a multiplication of the Gaussian distribution function and the prior probability is modeled by Markov random field (Eq. 3).

$$
\begin{aligned}
& P_{X_{s} \mid Y}\left(x_{s} \mid y, \theta\right)= \\
& \sum_{X \in \Omega_{k, s}} P_{X \mid Y}(x \mid y, \theta) \propto \sum_{X \in \Omega_{k, s}}\left\{\left[\prod_{i=1}^{N} \frac{1}{\sqrt{2 \pi \sigma_{x_{i}}^{2}}}\right] \cdot \exp \left(-\sum_{i=1}^{N} \frac{\left(y_{i}-\mu_{x_{i}}\right)^{2}}{2 \sigma_{x_{i}}^{2}}-\sum_{\{r, s\} \in c} \frac{1}{T(n)} \delta\left(x_{r}, x_{s}\right)\right)\right\}
\end{aligned}
$$

In Eq. (3), $N$ is the total number of voxels, $\left(\mu_{x i}, \sigma_{x i}^{2}\right)$ are model parameters of class $x_{i}, y_{i}$ is the intensity of a voxel, $T(n)$ is called temperature, and $\delta\left(x_{\mathrm{r}}, x_{s}\right)$ is a function that contributes to the labels of the neighboring voxels $(\mathrm{r})$ when determining the label of $s$. For all neighboring voxels whose labels $\left(x_{r}\right)$ same as $x_{s}$, the output of the weighting function is zero. Otherwise, a value of 0.5 or 1 is assigned according to Fig. 3. In our method, we consider six neighboring voxels; four in the same slice and two in the upper and the lower slices (Fig. 4.)

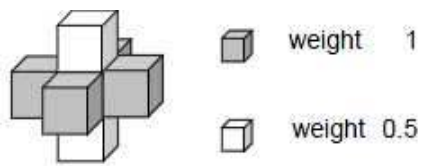

Fig. 4. Weight functions for 6-neighbours of a voxel.

Optimization of Eq. (2) is not simple. We use the simulated annealing method to iteratively determine an estimate [9], which is given by $T(n)=T_{1} / \log (n+c)$. Here, $T_{1}$ is the initial temperature which is a large constant, $c$ is a constant number and $n$ is the iteration number. After determining the MPM estimate (the E-step), the model parameters are calculated (the M-step). We continue the iteration of the two steps until convergence is achieved. As a result, we obtain tumor candidates that may include both true and false positive regions. In our experiments, we assign the values of 1.4 to $c, 50$ to $n$, and 2.0 to $T_{1}$ through several testing runs. To remove false results, we use the shape information described below.

\section{Candidate selection with shape filter}

The tumor candidates, which were detected in the previous section, include many false positives because the detection used only intensity information. Therefore, in this step, we perform a selection process using a shape filter. We define the following five evaluation 
criteria: (1) size, (2) shape of each slice, (3) regional variation among the slices, (4) location in the image, and (5) numbers of connectivity among the slices.

In this study, we assume that the shape of a tumor is approximately spherical. Therefore, for the second criteria, we use a shape filter as shown in Fig. 5. For each tumor candidate, we first find a center of gravity, and then calculate distances from it to the 12 points which are on the edge of tumor candidate as shown in Fig.5. 4 points are cross points with the bounding box (line of a rectangle), which are shown in Fig. 5 as diamond points. 8 points are boundary points sampled at intervals of 45 degree, which are shown in Fig. 5 as small circle points. The ratio of maximum distance and minimum distance is used as a measure of shape. The ratio is a value larger than or equal to 1 . If the candidate's shape is like a circle, the ratio will be 1 . Since the tumor is considered having a spherical shape, the candidates are rejected if their ratio is larger than a pre-defined threshold (in our research, the threshold is set as 4$)$.

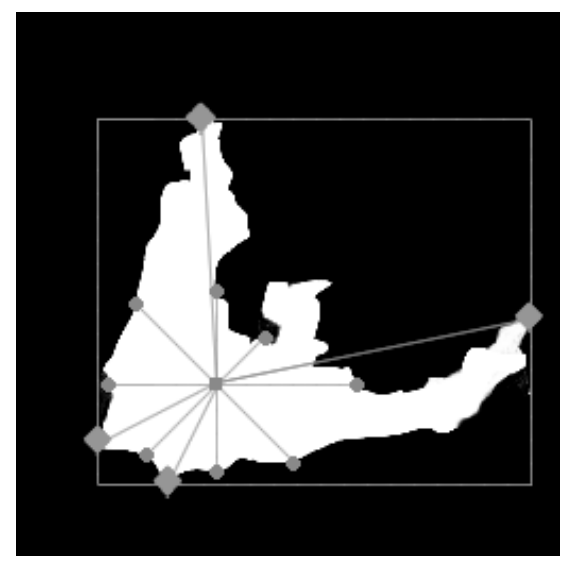

Fig. 5. Illustration of a shape filter

Though most false positive candidates can be rejected by the use of above 5 criterions, some tumor points will also be rejected. In order to recover the rejected true tumors, we use a new criterion, which is shown in Fig. 6 to check the rejected candidates. For each rejected tumor candidate, we first generate an edge image, and then superimpose it with two circles $L_{\text {in }}$ and $L_{\text {out }}$ having radius $r_{\text {in }}$ and $r_{\text {out }}$, respectively, and centers corresponding to the center of the tumor candidate's 2D ROI. Their parameters are defined in Eq. 4.

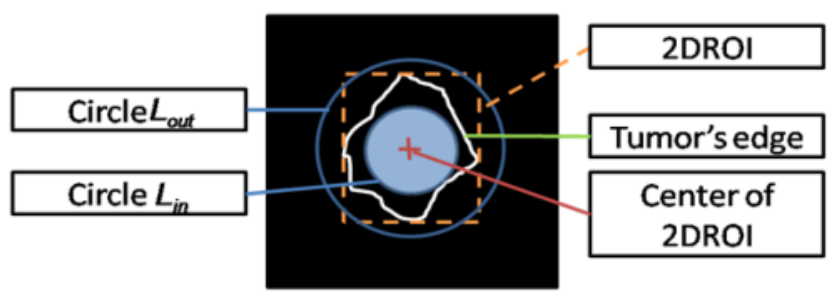

Fig. 6. Illustration of a shape filter for recheck 


$$
\left\{\begin{array}{c}
r_{\text {out }}=4+L / 2, \quad r_{\text {in }}=r_{\text {out }} / 2, \quad \text { if }(L \geq 10) \\
r_{\text {out }}=2+L / 2, \quad r_{\text {in }}=r_{\text {out }} / 2-1, \text { if }(L<10)
\end{array}\right.
$$

Here, $L$ is the longer side length of $2 \mathrm{D}$ ROI. If the shape of a tumor candidate is approximately spherical, then a major portion of the tumor region is bounded by the circle $L_{\text {in }}$ and the edge of the tumor is between the circles $L_{\text {in }}$ and $L_{\text {out }}$.

\section{Experimental results}

We applied our proposed method to five sets of CT images. Information on each image is shown in Table 1. Data sets 1, 2, and 3 were used in the JAMIT CAD contest in July 2010, while Data sets 4 and 5 were used in the MICCA Liver Tumor Segmentation Challenge 2008 [11]. Table 2 shows the results of tumor detection. In this study, if a part of a tumor is detected in the correct region, we consider the result as a true positive. Because only the ground truth for Data sets 1,2, and 3 were known, Table 2 shows comparisons between the detected results and the ground truth for Data sets 1-3. The proposed method provides accurate detection results for Data sets 1 and 2. For Data set 3, the detection rate is about $50 \%$ because the image includes numerous minute tumors.

\begin{tabular}{|c|c|c|c|c|c|c|}
\hline \multirow{2}{*}{ Data set } & \multicolumn{3}{|c|}{ Size } & \multicolumn{3}{c|}{ Spacing } \\
\cline { 2 - 7 } & $\mathrm{x}$ & $\mathrm{y}$ & $\mathrm{z}$ & $\mathrm{x}$ & $\mathrm{y}$ & $\mathrm{z}$ \\
\hline 1 & 512 & 512 & 156 & 0.625 & 0.625 & 1.00 \\
\hline 2 & 512 & 512 & 191 & 0.732 & 0.732 & 1.00 \\
\hline 3 & 512 & 512 & 200 & 0.72 & 0.72 & 1.00 \\
\hline 4 & 512 & 512 & 173 & 0.59 & 0.59 & 1.50 \\
\hline 5 & 512 & 512 & 172 & 0.77 & 0.77 & 1.50 \\
\hline
\end{tabular}

Table 1. Information on each dataset

\begin{tabular}{|c|c|c|c|}
\hline Data set & False negative & True Positive & Actual number \\
\hline 1 & 0 & 2 & 2 \\
\hline 2 & 0 & 5 & 5 \\
\hline 3 & 7 & 6 & 13 \\
\hline
\end{tabular}

Table 2. Number of detected tumors

Fig. 7 shows the results of tumor detection using the EM/MPM algorithm and the method based on [6]. It removed high intensities without employing Maximum likelihood method and applied the EM algorithm. The results show that our proposed method is superior to the previous one. The reason is considered to be the use of histogram transformation with PDFs. In the previous method, the EM algorithm took more time to converge compared with that in our proposed method; this was because the proposed method employs Maximum likelihood method. Moreover, irregular shapes could be removed by using the shape filter [Fig. 7(d)]. 

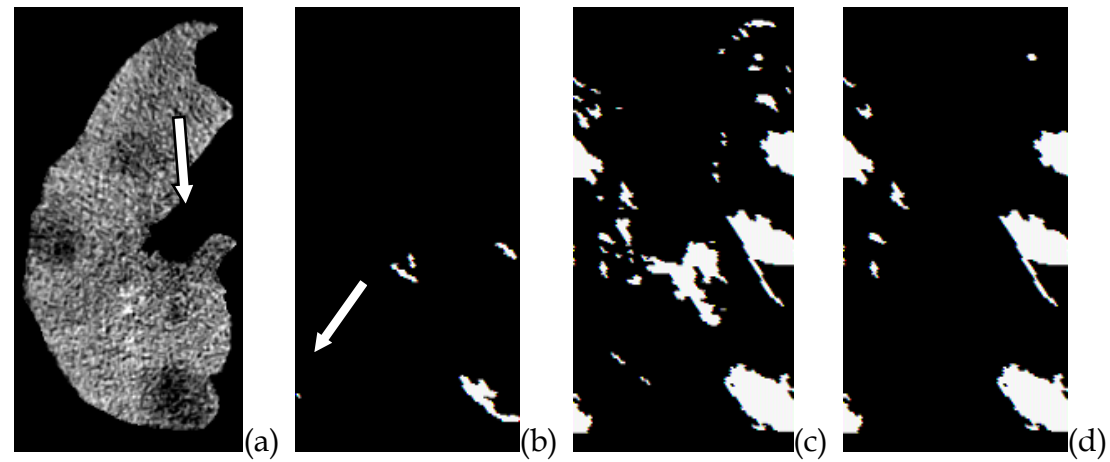

Fig. 7. (a) the original image (Data set 4). The tumor detection result obtained (b) using the method based on [6], (c) the proposed method, and (d) by the application of a shape filter to the image in (c). (In (b)-(d), the detected regions are white and the arrows indicate the locations of the detected tumors.)

Fig. 8 show the results of the experiments for Data set 3. We used four methods: EM with and without preprocessing (contrast enhancement) and EM/MPM with and without preprocessing. As we used different pre-processing for EM and EM/MPM, it may affect the result a little. However, Figures 8(c)-(f) are the images obtained after morphology operations. Figures $8(\mathrm{c})$, (d) demonstrate the effectiveness of our histogram transformation. Comparing Figs. 8(c), (e) with Figs. 8(d), (f), we find that using EM/MPM improves performance.
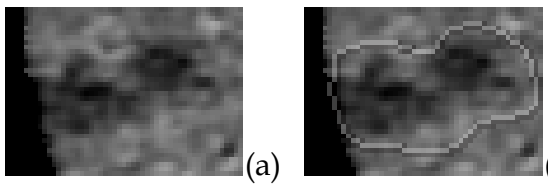

(b)
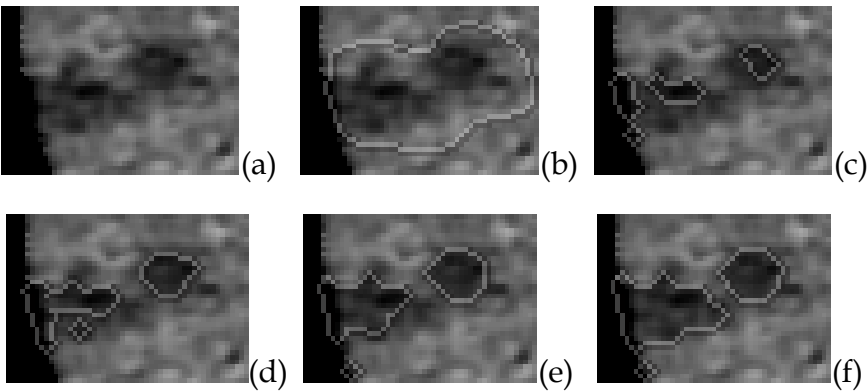

Fig. 8. Results after morphology (white lines) (a) Smoothed original image (b) answer (c) EM without preprocessing (d) EM with preprocessing (e) EM/MPM without preprocessing (f) EM/MPM with preprocessing

Next, we quantitatively evaluate the tumor segmentation performance in terms of the metrics proposed in the MICCAI Liver Tumor Segmentation Challenge 2008 [11]. The metrics are the volumetric overlap error (Overlap Error), absolute relative volume difference (Vol. dif.), average symmetric surface distance (Ave. Dist.), RMS symmetric surface distance (RMS Dist.), and maximum surface distance (Max. Dist.). For ideal segmentation, all metrics should be zero. Table 3 shows the results obtained for one slice of a segmented region in a tumor by the metrics given in [11]. For Data set 1, regions in which tumors are detected are not solely represented by dark regions but also by bright voxels around them. Our proposed method can detect dark tumor regions; however, it cannot detect the bright tumor regions. Therefore, we 
excluded the results for Data set 1 and included only the results for the other data sets. We compared the current method with the previous method on the basis of the abovementioned method [6]. It is obvious from the results that we have improved on all metrics.

\begin{tabular}{|c|c|c|c|c|c|c|}
\hline \multirow{2}{*}{$\begin{array}{c}\tilde{\tau} \\
\tilde{\sigma}\end{array}$} & Method & $\begin{array}{c}\text { Overlap } \\
\text { Error } \\
{[\%]}\end{array}$ & $\begin{array}{c}\text { Vol. dif. } \\
{[\%]}\end{array}$ & $\begin{array}{c}\text { Ave } \\
\text { Dist. } \\
{[\mathrm{mm}]}\end{array}$ & $\begin{array}{c}\text { RMS } \\
\text { Dist. } \\
{[\mathrm{mm}]}\end{array}$ & $\begin{array}{c}\text { Max. } \\
\text { Dist. } \\
{[\mathrm{mm}]}\end{array}$ \\
\hline \multirow{2}{*}{2} & previous & 74.27 & 68.09 & 1.28 & 1.84 & 5.00 \\
\cline { 2 - 7 } & proposed & 59.54 & 50.92 & 0.84 & 1.45 & 5.00 \\
\hline \multirow{2}{*}{3} & previous & 75.63 & 72.08 & 2.76 & 4.20 & 13.45 \\
\cline { 2 - 7 } & proposed & 66.43 & 61.29 & 1.84 & 3.08 & 11.18 \\
\hline \multirow{2}{*}{4} & previous & 84.29 & 84.29 & 4.84 & 6.57 & 16.27 \\
\cline { 2 - 7 } & proposed & 16.87 & 8.45 & 0.17 & 0.65 & 5.00 \\
\hline \multirow{2}{*}{5} & previous & 6.71 & 4.53 & 0.04 & 0.28 & 4.24 \\
\cline { 2 - 7 } & proposed & 5.82 & 1.94 & 0.04 & 0.24 & 3.60 \\
\hline
\end{tabular}

Table 3. Performance comparisons

\section{Conclusions}

We have proposed a new method to detect tumors automatically in CT image. By using contrast enhancement with PDFs of different tissue classes in a newly devised histogram transformation method, we can enhance the image contrast. Moreover, by using the EM/MPM algorithm, we can detect tumors more accurately. We plan to improve our work to handle the large morphology variation of tumors.

\section{Acknowledgement}

We thank associate professor Akinobu Shimizu of Tokyo University of Agriculture and Technology for providing liver region extract program. We also thank JAMIT and MICCAI for providing CT images. This work was supported in part by the Grant-in Aid for Scientific Research from the Japanese Ministry for Education, Science, Culture and Sports under the Grant No. 21300070 and 22103513, and in part by the Research fund from Ritsumeikan Global Innovation Research Organization (R-GIRO). Wei Xiong and Jiayin Zhou acknowledge supports from Singapore A*STAR project JCOAG03_FG05_2009.

\section{References}

[1] Center for Cancer Control and Information Services, National Cancer Center, Japan, http://ganjoho.jp/public/statistics/pub/statistics01.html 
[2] D. Smeets, D. Loeckx, B. Stijnen, B. De Dobbelaer, D. Vandermeulen, P. Suetens, Semiautomatic level set segmentation of liver tumors combining a spiral scanning technique withsupervised fuzzy pixel classification, Medical image analysis, vol. 14, no. 1, pp. 13-20, February 2010.

[3] Häme, Y., Alhonnoro, T., Pollari, M.: Image Analysis for Liver Tumor Ablation Treatment Planning, Hands-on Image Processing 2009, Robotiker-Tecnalia.

[4] K. Mala, V. Sadasivam, S. Alagappan, "Neural Network Based Texture Analysis of Liver Tumor from Computed Tomography Images, " International Journal of Biomedical Sciences 2, 33-40, 2006.

[5] Seung-Jin Park, Kyung-Sik Seo, Jong-An Park: "Automatic Hepatic Tumor Segmentation Using Statical Optimal Threshold", Computational Science - ICCS 2005, Springer Berlin / Heidelberg, Volume 3514, pp 934-940, 2005.

[6] Y. Masuda, A. H. Foruzan, T. Tateyama, Y. W. Chen, "Automatic liver tumor detection using EM/MPM algorithm and shape information ", IEICE technical report 110(28), 25-30, 2010-05-13

[7] Y. Masuda, A. H. Foruzan, T. Tateyama, Y. W. Chen, "Automatic liver tumor detection using EM algorithm and 3DROI," Kamsao-section Joint Convention of Institutes of Electrical Engineerin, G310, 2009.

[8] A. Shimizu, http://www.tuat.ac.jp/ simizlab

[9] M.L. Comer and E. J. Delp. The EM/MPM Algorithm for Segmentation of Textured Images: Analysis and Further Experimental Results, IEEE Transactions on Image Processing. , 9 (10) 1731-1744 October 2000.

[10] J.L. Marrquin, S. Mitter and T. Poggie. Probabilistic Solution of Ill-Posed Problems in Computational Vision, Journal of American Statistical Association, 28 (397) 76-89 March 1987.

[11] X. Deng and G. Du:"Editorial: 3D Segmentation in the Clinic: A Grand Challenge II Liver Tumor Segmentation", http:/ / grand-challenge2008.bigr.nl/proceedings/liver/articles.html 


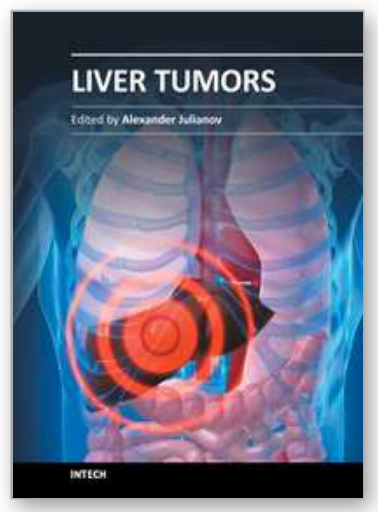

\author{
Liver Tumors \\ Edited by Prof. Alexander Julianov
}

ISBN 978-953-51-0036-2

Hard cover, 200 pages

Publisher InTech

Published online 03, February, 2012

Published in print edition February, 2012

This book is oriented towards clinicians and scientists in the field of the management of patients with liver tumors. As many unresolved problems regarding primary and metastatic liver cancer still await investigation, I hope this book can serve as a tiny step on a long way that we need to run on the battlefield of liver tumors.

\title{
How to reference
}

In order to correctly reference this scholarly work, feel free to copy and paste the following:

Yu Masuda, Tomoko Tateyama, Wei Xiong, Jiayin Zhou, Makoto Wakamiya, Syuzo Kanasaki, Akira Furukawa and Yen Wei Chen (2012). Liver Tumor Detection in CT Images by Adaptive Contrast Enhancement and the EM/MPM Algorithm, Liver Tumors, Prof. Alexander Julianov (Ed.), ISBN: 978-953-51-0036-2, InTech, Available from: http://www.intechopen.com/books/liver-tumors/liver-tumor-detection-in-ct-images-by-adaptive-contrastenhancement-and-the-em-mpm-algorithm

\section{INTECH}

open science | open minds

\author{
InTech Europe \\ University Campus STeP Ri \\ Slavka Krautzeka 83/A \\ 51000 Rijeka, Croatia \\ Phone: +385 (51) 770447 \\ Fax: +385 (51) 686166 \\ www.intechopen.com
}

\author{
InTech China \\ Unit 405, Office Block, Hotel Equatorial Shanghai \\ No.65, Yan An Road (West), Shanghai, 200040, China \\ 中国上海市延安西路65号上海国际贵都大饭店办公楼 405 单元 \\ Phone: +86-21-62489820 \\ Fax: $+86-21-62489821$
}


(C) 2012 The Author(s). Licensee IntechOpen. This is an open access article distributed under the terms of the Creative Commons Attribution 3.0 License, which permits unrestricted use, distribution, and reproduction in any medium, provided the original work is properly cited. 